\section{Synthesis and Spectral Characterization of New Aromatic Random Copolyester}

\section{G. Annamalai ${ }^{1}$, R. Subramanian ${ }^{2}$, S. Guhanathan ${ }^{3}$, G. Elango ${ }^{1}$}

${ }^{1}$ Department of Chemistry, Government Arts College, Tiruvannamalai, Tamil Nadu, India, ${ }^{2}$ Department of Chemistry, Sun Arts and Science College, Tiruvannamalai, Tamil Nadu, India, ${ }^{3}$ Department of

Chemistry, Muthurangam Government Arts College, Vellore, Tamil Nadu, India

\section{ABSTRACT}

Copolyesters are one of the most significant classes of polymers with repeating carboxylate group in their backbone. A novel random copolyester was synthesized using 1,4-dihydroxy naphthalene, quinol with phthaloyl chloride as dissimilar diols and diacid chloride monomers. The obtained polymers were characterized by ultraviolet-Visible spectroscopy (UV-Vis), Fourier transform spectroscopy (FTIR), nuclear magnetic resonance (NMR), thermogravimetric analysis, and differential scanning calorimeter. The surface morphology and crystalline nature of the polymers were analyzed using scanning electron microscope (SEM) and polarizing optical microscope. From the above, all details the synthesized polyesters are novel to possess the nature of liquid crystalline. Results of the exploration revealed that the innovative entries of copolymer were recommended into the set of liquid crystalline copolymers.

\section{INTRODUCTION}

Liquid crystalline polymers, counting main-chain and sidechain types, have been the subjects of several publications ${ }^{[1-3]}$ and review articles. ${ }^{[4]}$ Liquid crystals (LC) are well conservative in basic research as well as in the development of commercial use. For example, usually nematic LC are used for display appliance applications. In general, the liquid crystalline polymer combines mesogenic units and high molecular weight, and so exhibits admirable anisotropic physical properties as possessing and suitable molecular couture. Among them, the wholly aromatic liquid crystalline polyesters have generally received significant importance for industrial applications, Since of their use in high temperatures and comparatively high glass transition temperatures. ${ }^{[5]}$ These polymers show liquid crystalline manners similar to their inventor monomers, but with higher stability. ${ }^{[6]}$ Meanwhile, main-chain liquid crystalline polymers synthesized from bifunctional monomers also show liquid crystalline behavior similar to those of low mass model compounds. Above the glass transition temperature ( $\mathrm{Tg}$ ), segments in polymer chains can liberally move due to the micro-Brownian movement, and the orientation of decoupled mesogens induces optical anisotropy, which is a feature of the liquid crystalline compounds. Cross-linking between the polymer chains results in the network polymers and cross linking polymers. In this case, macro-Brownian movement, i.e., the motion of entire molecules, is prohibited by crosslinking, but micro-Brownian motion of segments is basically unaffected. Theoretically, LC polymers could be cross-linked to form elastomers in which properties arising from the liquid crystalline behavior could be really immobilized, yielding anisotropic solids with strange properties. Therefore, it is potential to realize a form-retaining liquid crystalline material preferred shape. The liquid crystalline polymers with rubber elasticity, which have been the subject of a number of reviews, can be industrial as a promising functional material. ${ }^{[7-12]}$ The anisotropic network can be formed through cross-linking from different liquid crystalline polymers, which consist of main-chain, side-chain, and combined polymers with reactive groups. During the past two decades, significant evolution has been made to enlarge side-chain LC polymers as materials for electric-optics, integrated optics, and storage devices. For all of these applications, it is essential to have samples with the optical axes of the mesogens being macroscopically and regularly aligned so that the LC polymers function as single crystals optically. Under an external field, consistently 
aligned mesogenic monomers containing two reactive groups or prepolymers incorporating reactive groups can be photo- or thermo-polymerized or cross-linked by one or two steps with the addition of a non-mesogenic cross-linking agent. As a result, macroscopically aligned elastomers named as liquid single crystal elastomers and anisotropic LC networks with different cross-link densities can be prepared, with macroscopic orientation in the liquid crystalline state fixed in the solid sample. Anisotropic LC gels (ALCGs) consist of a swollen anisotropic network and noncovalently bonded, but strongly oriented domains of low molar mass LC. During the past decade, the research in this field was very dynamic and fascinating, especially on their potential applications as intriguing, smart materials for converting electrical or optical energy into mechanical energy and vice versa, such as artificial muscles, light scattering electro-optical switches, and display materials, electro- or photo-controllable micro- or nanomachinery, electrically switchable color-tunable reflectors and full-color reflective displays, fine-tunable and low-threshold mirror-less lasing, etc. The main objective of this article is to review the research progress in the field of liquid crystalline polymers. A new technological application of liquid crystalline polymer has recent attention for their good thermal stability, excellent mechanical properties. ${ }^{[13]}$ The texture, mechanical strength, and thermal stability of the polymer were completely dependent on the chemical structure and molecular weight of the monomers, thus fully aromatic monomers show good liquid crystalline property. ${ }^{[14]}$ In the past decades, many researchers have developed many LCP's with considerable efforts on modification of the monomeric structure to reach more practical usage in industries. ${ }^{[15-17]}$

Incorporation of aromatic monomers to the polymer forms a rigid structure in the main chain of the polymer and also increase the melting, decomposition temperature, and mechanical strength and more of LCP's. ${ }^{[18]}$ Number of methods used to synthesize the polyester but the simple and the best method to synthesis LCP's is direct polycondensation, ${ }^{[19,20]}$ because this direct polycondensation technique was simple to prepare LCP's without catalyst and other chemicals. ${ }^{[21]}$

In this work, we describe the synthesis of novel LCP's using quinol using direct polycondensation reaction in the presence of 1, 4-dihydroxy naphthalene and phthaloylchloride. To the best of our knowledge, this is the first scheme of synthesis of copolyester at reasonable reaction condition.

\section{MATERIALS AND METHODS}

Chemicals of high purity were obtained from a variety of commercial sources, phthaloylchloride, quinol, 1, 4-dihydroxy naphthalene all purchased from Merck, Mumbai, India.

Decontamination of solvent used in this process was 1, 2-dichlorobenzene, kept overnight in anhydrous calcium chloride then filtered and purified using fractional distillation method at the temperature of $180^{\circ} \mathrm{C}$ and finally separated. Petroleum ether was dried in the presence of anhydrous calcium chloride and finally distilled. Other solvents such as acetone, chloroform, carbontetra chloride, o-chlorophenol, dimethyl sulfoxide, and methanol all in AR grade were used to ensure the solubility of the polymer.

\section{Experimental Method}

Synthesis of Poly(4-oxynaphthalen-1-yl (4-oxyphenyl)Phthalate

Direct polycondensation of diols with diacid chloride phthaloyl chloride, quinol, 1,4-dihydroxy naphthalene in the ratio of $2: 3: 1$ taken in a reaction flask with $250 \mathrm{ml}$ of 1,2 -dichlorobenzene and refluxed at $130-150^{\circ} \mathrm{C}$ at $36 \mathrm{~h}$ pursed in a nitrogen atmosphere with constant stirring. The content was cooled and dissolved in petroleum ether and kept in the refrigerator for overnight. Filtered crude was dissolved in acetone and evaporated to get pure polyester, and again it dried in a vacuum with phosphorous pentoxide. The yield of the polymer obtained was $81 \%$.

\section{Characterization of random copolyesters}

The Fourier transform spectroscopy (FTIR) spectrum of copolyester was reported using Perkin-Elmer spectrophotometer. FTIR spectrophotometer with the samples incorporated in $\mathrm{KBr}$ pellets. The ultraviolet (UV)-Visible spectral analysis was performed on Shimadzu-uv-160A spectrophotometer using acetone solution. The ${ }^{1} \mathrm{H}$ Nuclear magnetic resonance (NMR) spectra were recorded using JEOL GSX 400 FT-NMR spectrometer operating at room temperature. Samples for analysis were prepared by dissolving about $10 \mathrm{mg}$ of the copolyester in $5 \mathrm{ml}$ of spectral grade $\mathrm{CDCl}_{3}$ solvent. Gel permeation chromatography (GPC) calibration curves were recorded with waters associates liquid chromatography (Waters 510 high-performance liquid chromatography [HPLC] pump, Waters 717 autosampler). Polystyrene standard was used for calibration. Tetrahydrofuran eluent was used (flow rate $=1.0 \mathrm{ml} / \mathrm{min}$ ). Higher magnification pictures were recorded for all copolyesters with scanning electron microscope (SEM) [Figures 5 and 8].

\section{RESULTS AND DISCUSSION}

\section{Solubility}

Copolyester, $10 \mathrm{mg}$ was taken in a small stopper test tube which contains $5 \mathrm{ml}$ of the solvent and kept for $24 \mathrm{~h}$ with infrequent shaking. Since, the polyester was insoluble in the cold it was slowly heated up to boiling point of the solvent. Thus, the polymer was dissolved. The process was done with different solvent qualitatively.

\section{FTIR analysis}

The FTIR spectroscopy is one of the important tools to identify the functional groups present in the synthesized polyester P6NPQ. The sample was scanned and recorded in the range of $4000-400 \mathrm{~cm}^{-1}$. The characteristic absorption bands obtained for Co-polyester are shown in Figure 2. A peak obtained at $1720.03 \mathrm{~cm}^{-1}$ arising from free and associated carbonyl stretching of the aromatic-aromatic ester group in the polymer [Figure 1].

The bands observed $1192.03 \mathrm{~cm}^{-1}$ corresponding to the stretching vibrations of the ester $\mathrm{C}-\mathrm{O}$. The band arising from characteristic absorptions of naphthalene moiety was present in $3328.26 \mathrm{~cm}^{-1}$. A broad peak observed at $3776.39 \mathrm{~cm}^{-1}$ reveals the $p$-substituted aromatic ring present synthesized polyester P6NPQ. ${ }^{[22]}$ 


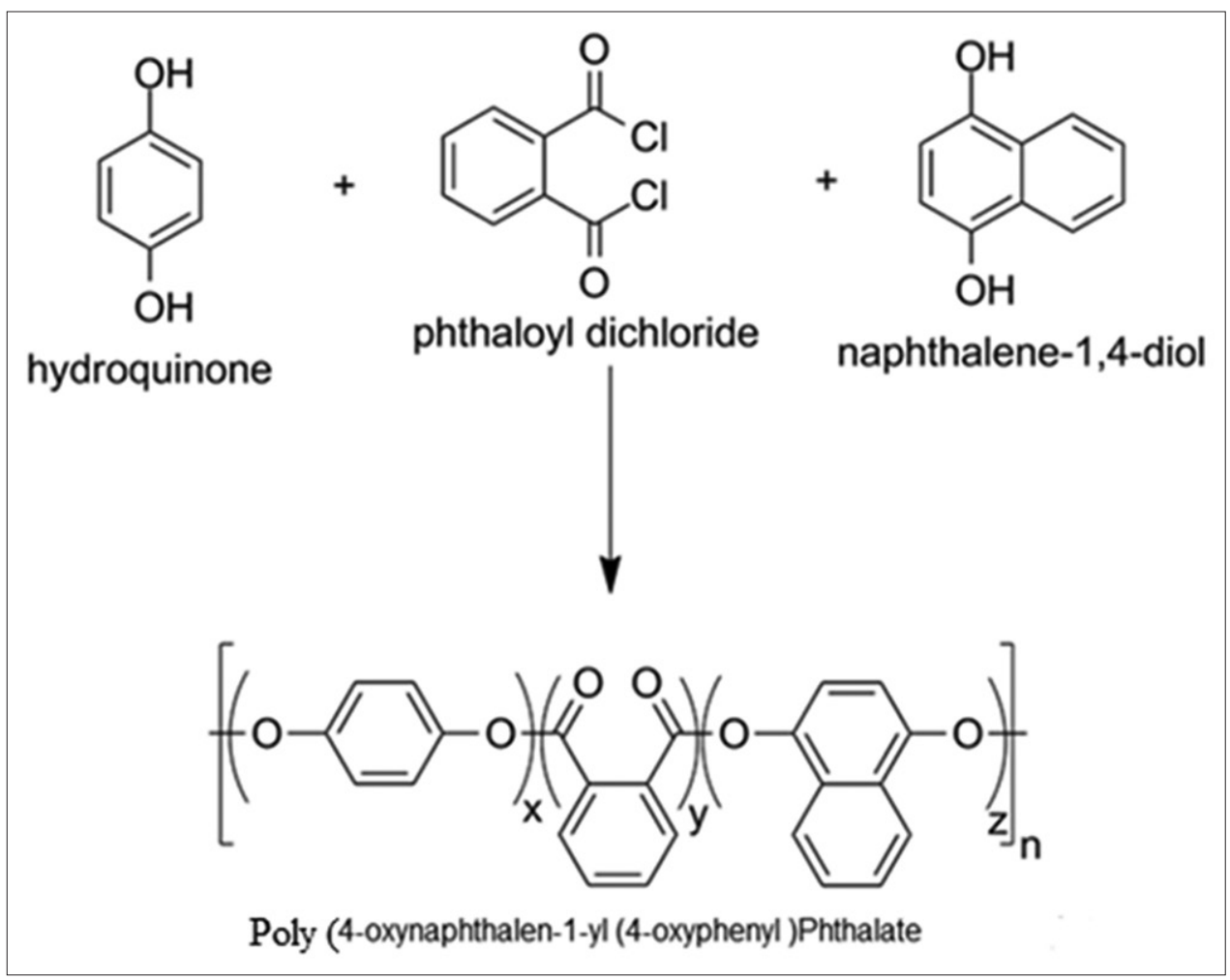

Figure 1: Synthesis of polymer

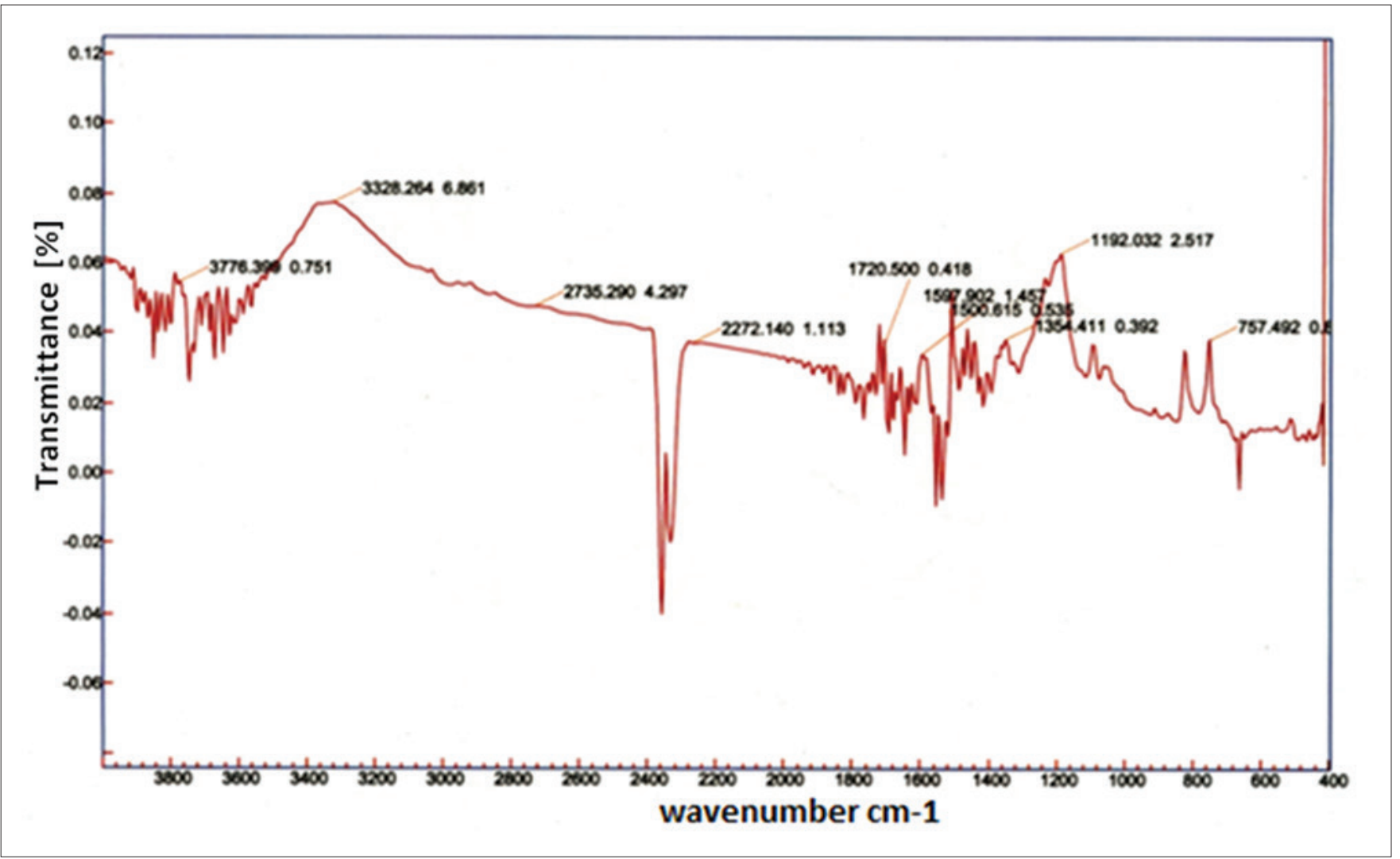

Figure 2: FTIR Spectrum of P6NPQ 


\section{UV-visible spectra}

Synthesized arbitrary copolyester reported in the current study contains substituted benzene chromophore. Therefore, there are two possible electronic transitions, namely $\pi \rightarrow \pi^{*}$ and $\mathrm{n} \rightarrow \pi^{*}$. The UV-Visible spectrum of the polyester was recorded in acetone, and it is found to be similar with two absorption maxima in each of these copolyesters. The spectrum of polyester is indulged in Figure 3. It is noted that the absorption maxima is not influenced by the length of the spacer in the polymer chain, but subjective by the number of condensed rings present in them.

\section{NMR SPECTRAL ANALYSIS}

NMR spectroscopy was used to identify the formation of P6NPQ. The sample was analyzed with a Bruker $300 \mathrm{MHz}$ spectrometer working at room temperature. Spectrum was recorded in $\mathrm{CDCl}_{3}$, tetramethylsilane (TMS) as an internal standard. ${ }^{[23]}$ Chemical shifts values were reported in ppm relative to the signal of TMS. ${ }^{1} \mathrm{HNMR}$ spectrum of polyester is shown

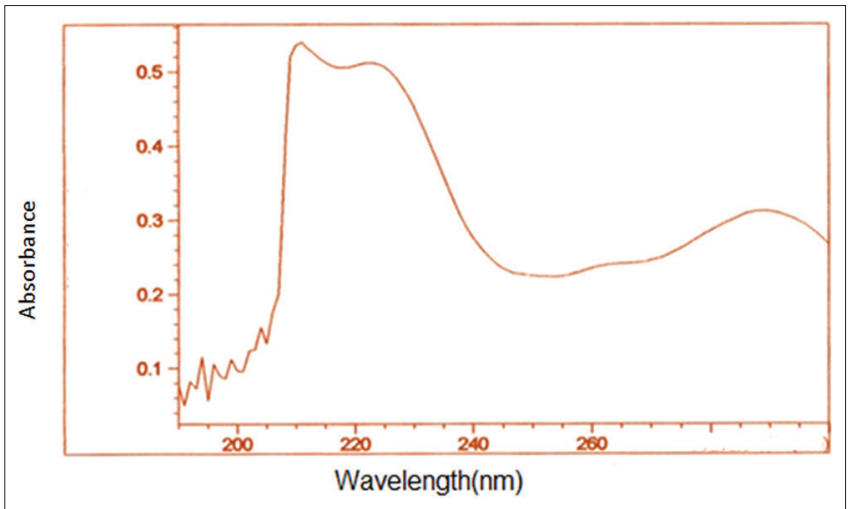

Figure 3: Ultraviolet Spectrum of P6NPQ in Figure 4. The peak positions ( $\delta$ values) in this spectrum are presented in Table 1 . The chemical shift values can be explained on the basis of structural units present in these polyesters. The ring protons of phthaloyl moiety are one doublet and one triplet are highly deshielded and hence have high d values. Similarly, the protons of 1, 3-phenylene and 1, 4-phenylene rings present in the polymer chain also absorb at downfield in 1,4-naphthalene unit appear one singlet, one doublet, and one triplet nature.

\section{GPC and HPLC study}

A newer technique involves GPC, a qualified method that yields the absolute molecular weight distribution in about half an hour per sample. This method also called as size exclusion chromatography. GPC has its supreme value for measuring the molecular weight and polydispersity of synthetic polymers. A strongly correlated method, HPLC is more useful for polymers containing functional groups. ${ }^{[24]}$ Both of these methods depending on the distribution coefficients, relating the selective distribution of an analyte between the mobile phase and stationary phase.

\section{Thermo Gravimetric Analysis (TGA)}

The thermal stability of the copolyesters was determined by TGA. It can be also used to determine the kinetic parameters of degradation of the copolyester. The continuous weight loss curve for the thermal degradation of the copolyester is given in Figure 6. The degradation of all copolyester was carried out in a nitrogen atmosphere at a heating rate of $20^{\circ} \mathrm{C} / \mathrm{min}$. This

Table 1: ${ }^{1} \mathrm{H}$ NMR data for P6NPQ

\begin{tabular}{ll}
\hline Chemical shift $(\boldsymbol{\delta}) \mathbf{( p p m )}$ & Type of protons \\
\hline 2.32 & Protons of the ester group \\
$6.92-7.47$ & Aromatic protons \\
$6.5-8.0$ & 1,2 -phenylene, 1,4-phenylene \\
\hline
\end{tabular}

NMR: Nuclear magnetic resonance

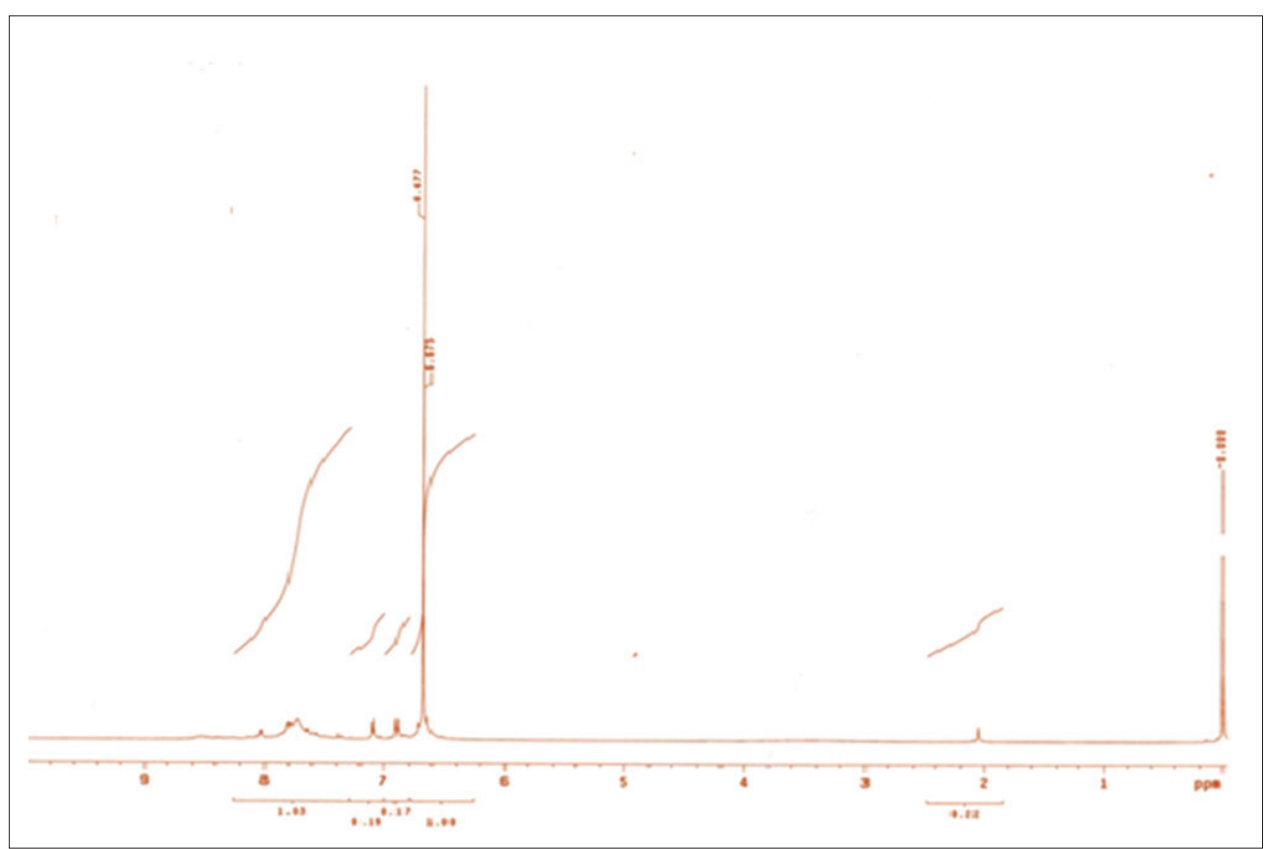

Figure 4: ${ }^{1} \mathrm{H}$ Nuclear magnetic resonance spectrum of P6NPQ 
thermogram suggests that the copolyester is thermally stable up to $100^{\circ} \mathrm{C}$. There are three steps in the degradation. The first degradation step starts around $100-225^{\circ} \mathrm{C}$, and about $10 \%$ of the copolyester was degraded in this step. The second stage of degradation starts around at $225-350^{\circ} \mathrm{C}$, and in the state, approximately $60 \%$ of the copolyester gets degraded. The third stage of degradation shows that $70 \%$ of the polyester was degraded in the temperature range $400^{\circ} \mathrm{C}-600^{\circ} \mathrm{C}$ except for P6NPQ which shows $70 \%$ degradation at $620^{\circ} \mathrm{C}$. This may be due do the presence of rigid rod and crankshaft mesogens.

\section{Differential Scanning Calorimeter (DSC)}

DSC examination was carried out on a TA DSC Q100 calorimeter with a programmed heating procedure in a nitrogen atmosphere. The sample size was about $5 \mathrm{mg}$ and encapsulated in hermetically sealed aluminum pans, whose weight was kept constant. The temperature and heat flow scale at different cooling and heating rates were calibrated using standard materials such as indium and benzoic acid. ${ }^{[25]}$ P6NPQ Ther mogram contains sharp as well as broad peaks at characteristic temperatures indicating phase transitions, before melting. The transition temperatures were calculated from the DSC thermograms and they are presented in Figure 7. Along with the type of mesophase detected in a hot-stage polarizing microscope. ${ }^{[26]}$ These transition temperatures for the formation of mesophase determined by polarizing optical microscope (POM). These transition temperatures for the formation of mesophase were determined by POM and PXRD.

The $\mathrm{T}_{\mathrm{g}}$ and $\mathrm{Tm}$ of the polymers could be precisely

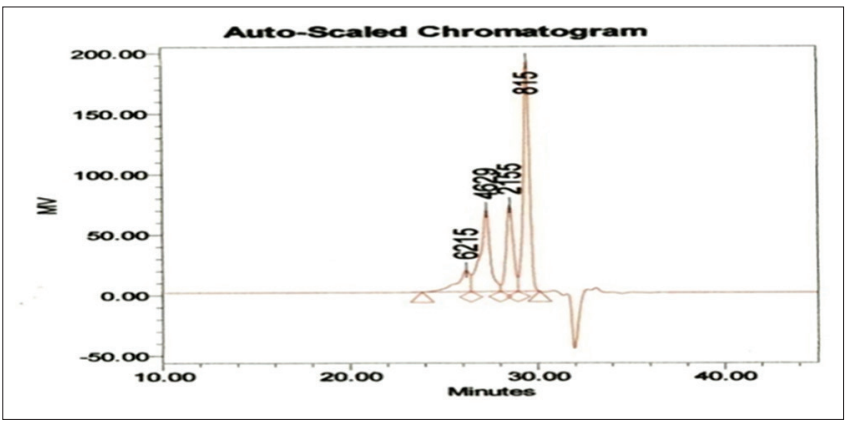

Figure 5: Gel permeation chromatography calibration curves

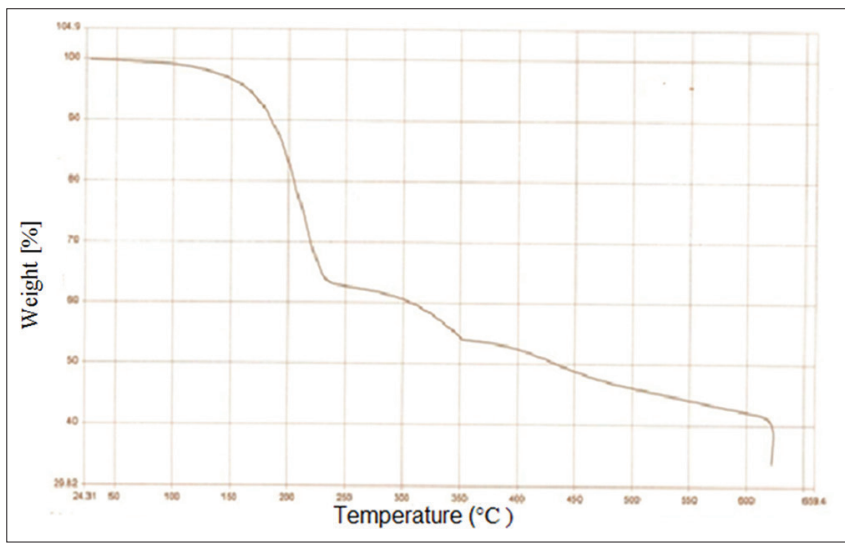

Figure 6: TGA Thermogram of P6NPQ random copolyester determined from heating curves of their differential scanning analysis in P6NPQ. This thermal behavior is due to the nature of the spacer which links the main chain. Polymers having high $\mathrm{T}_{g}$ and $\mathrm{Tm}$ contain rigid 1, 2-phenylene spacer. Polymers having low $\mathrm{T}_{\mathrm{g}}$ and $\mathrm{Tm}$ contain flexible spacer with serves to dissociate the disorder of the main chain from the greater order of the mesogenic groups and to decouple the motions of the mesogenic moiety from those of the polymer backbone. The effect of increased polymethylene flexible spacer length on the liquid crystalline polymer. ${ }^{[27]}$ P6NPQ is having same $\mathrm{T}_{g}$ and $\mathrm{Tm}$, containing same quinol and isomers of naphthalene diol. Copolyester having 1,4-naphthalene moieties show same transition temperature.

\section{Activation energies of degradation of the random copolyester}

The kinetics of thermal degradation of copolyester was considered by measuring the rate of modify in physical properties such as weight over a series of steady temperature. The activation energies [Table 2] are evaluated by Murray and White, Coats and Redfern as well as Doyle methods. ${ }^{[28-30]}$ In Murray and White method plots are plotted in between $\mathrm{T}^{-1} \times 10^{3}\left(\mathrm{~K}^{-1}\right)$ and $\ln [\ln (1-\mathrm{c})]-2 \ln \mathrm{T}$. In Coats and Redfern method plots are plotted in between $\mathrm{T}^{1} \times 10^{3}\left(\mathrm{~K}^{-1}\right)$ and $\log$ $\left[\ln (1-\mathrm{c}) / \mathrm{T}^{2}\right]$. In Doyle method plots are plotted in between $\mathrm{T}^{-1} \times 10^{3}\left(\mathrm{~K}^{-1}\right)$ and $\log [\ln (1-\mathrm{c})]$. The calculated activation energy values are given in Table 2 . Table 2 shows that the activation energies of the random copolyester are almost comparable. Relatively higher energy of activation values is observed in P6NPQ since the presence of 1, 4-phenylene and naphthalene moieties that have inbuilt stability factors due to ring structure and aromaticity.

\section{OPTICAL POLARIZING MICROSCOPIC STUDIES}

The characterization of LC by polarized light microscopy is mainly valuable for polymers possessing desired mechanical, thermal, and optical properties. Recently there has been considerable interest in TLC polyesters with low $\mathrm{Tg}$ values followed by a sharp transition to a mesophase with as a low Tm value as possible since these polymers are used in reprographic applications. The extensively employed technique to identify the mesophase in TLC polyesters is by optical polarizing microscopic investigation. ${ }^{[31]}$

Extensive investigation on low molecular mass LC has proved that LC state can be directly correlated with the chemical constitution of the molecules. ${ }^{[32-36]}$ Due to their chemical constitution, liquid crystalline phases having defined molecular organizations are formed.

\section{SEM studies}

The SEM micrographs are shown in Figure 9. The studies use the polyester surface. SEM investigation was carried out for polyester at diverse places on the surface of the film with changeable magnifications. As shown in Figure 9, P6NPQ polyester has extremely orientated and constant microstructure, which leads to excellent mechanical properties. Meanwhile, the polyester exhibited an identical distribution. ${ }^{[37]}$

14 Asian J Appl Res | 2019;5(1) 
Table 2: Calculation of activation energy for the degradation of P6NPQ

\begin{tabular}{|c|c|c|c|c|c|c|c|c|c|c|}
\hline \% Degradation & $\mathbf{T}(\mathbf{K})$ & $\begin{array}{c}\mathrm{T}^{-1} \times 10^{-3} \\
\mathrm{~K}^{-1}\end{array}$ & $\mathbf{1}-\mathbf{C}$ & $2 \ln T$ & $\mathbf{T}^{2}$ & $\begin{array}{c}\text { Ln } \\
(1-C)\end{array}$ & $\underset{[\ln (1-C)]}{\log }$ & $\stackrel{\operatorname{Ln}}{[\ln (1-C)]}$ & $\stackrel{\operatorname{Ln}}{[\ln (1-C)]-2 \ln T}$ & $\underset{\left[\ln (1-C) / T^{2}\right]}{\log }$ \\
\hline 10 & 462.10 & 2.1640 & 90 & 12.2715 & 213536 & 4.50 & 0.653 & 1.504 & -10.7675 & $3.2 \times 10^{-6}$ \\
\hline 20 & 482.90 & 2.0708 & 80 & 12.3596 & 233192 & 4.40 & 0.643 & 1.481 & -10.8786 & $2.8 \times 10^{-6}$ \\
\hline 30 & 496.10 & 2.0157 & 70 & 12.4135 & 246115 & 4.24 & 0.627 & 1.444 & -10.9695 & $2.3 \times 10^{-6}$ \\
\hline 40 & 506.00 & 1.9762 & 60 & 12.4530 & 256036 & 4.10 & 0.612 & 1.410 & -11.043 & $2.0 \times 10^{-6}$ \\
\hline 50 & 525.80 & 1.9018 & 50 & 12.5298 & 276465 & 3.90 & 0.591 & 1.361 & -11.1688 & $1.5 \times 10^{-6}$ \\
\hline 60 & 639.00 & 1.5649 & 40 & 12.9198 & 408321 & 3.70 & 0.568 & 1.308 & -11.6118 & $9.5 \times 10^{-7}$ \\
\hline 70 & 812.60 & 1.2306 & 30 & 13.4004 & 660318 & 3.40 & 0.531 & 1.224 & -12.1764 & $6.9 \times 10^{-7}$ \\
\hline 80 & 915.90 & 1.0918 & 20 & 13.6398 & 838872 & 3.00 & 0.477 & 1.099 & -12.5408 & $5.4 \times 10^{-7}$ \\
\hline
\end{tabular}

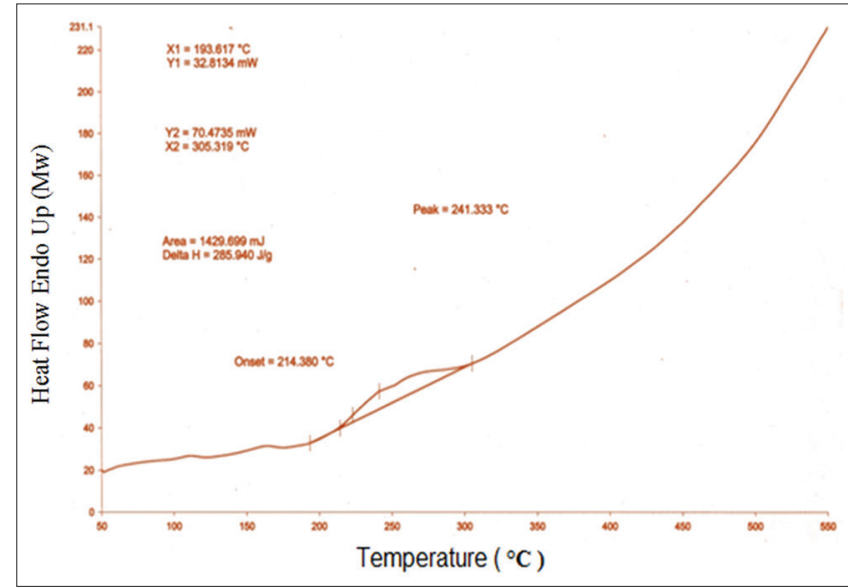

Figure 7: DSC Thermograms of P6NPQ

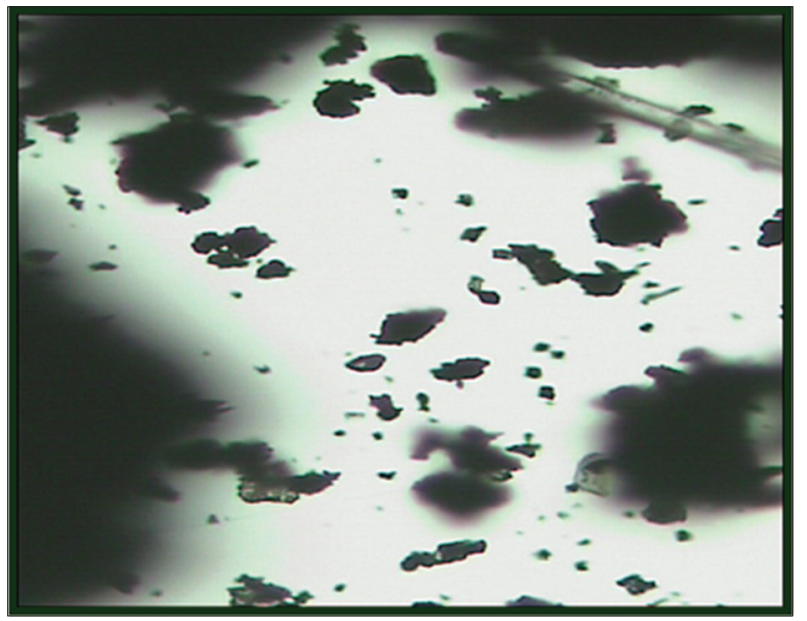

Figure 8: Optical polarized micrographs of P6NPQ

The representative SEM images of the condensed powder surfaces of polyester are shown in Figure 9. The rigid and long-range orientational order is apparent from the SEM micrographs of this polyester P6NPQ. It has been recommended that the microstructure of TLC polymeric materials has some evidence of crystallinity embedded within an extended chain structure of the mesophase. ${ }^{[38-43]}$ These crystallinities have been described as a non-periodic layer structure which propagates

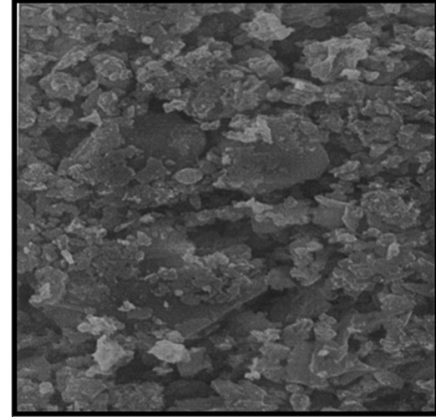

P6NPQ 15 kv ( SS50 X 100)

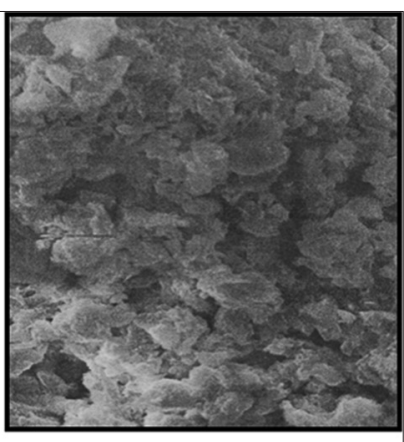

P6NPQ15 kv ( SS50 X 250)
Figure 9: Scanning electron microscope photomicrographs of P6NPQ

among adjacent oriented chains. The SEM photographs of these random copolyesters show such crystallinity on their film surface indicating long-range orientation order.

\section{CONCLUSIONS}

Synthesis of aromatic polyester with high molecular weight was done by simple polycondensation route followed by the characterization of complementary analytical techniques. The copolyester was soluble in acetone and chloroform. The chemical structure of the synthesized polyester was definite by FTIR, and ${ }^{1} \mathrm{H}$ NMR spectral values are in accordance with ester linkage and protons of the polymer. The thermal data, i.e., deprivation of the polyester were investigated that the liquid crystalline condition of the polyester was more proved by SEM micrograph of the LC polymer film provides useful information about film surface of the polymer structure. The thermotropic liquid crystalline arbitrary copolyester may be a potential applicant for future device technology.

\section{REFERENCES}

1. Jenkins AD, Kratochvil P, Suter UW. International union of pure glossaryof basic terms in polymer. Pure Appl Chem 2000;68:12.

2. Bruin P, Smedinga J, Pennings AJ, Jonkman MF. Biodegradable lysine diisocyanate based poly urethane network in artificial skin. Biomaterials 1990;11:291-5.

3. Rivero G, Nguyen L, Hillewaere XK, Du Prez FE. Remendable shape memory polyurethanes. Macromolecules 2014;47:2010-8.

4. Claeys B, Vervaeck A, Vervaet C, Remon JP, Hoogenboom R, De Geest BG, et al. Poly(2-ethyl-2-oxazoline) as matrix excipient for 
drug formulation by hot melt extrusion and injection molding. Macromol Rapid Commun 2012;33:1701-7.

5. Lorenzetti C, Finelli L, Lotti N, Vannini M, Gazzano M, Munari BC. Synthesis and characterization of poly(propylene terephthalate/2,6-naphthalate) random copolyesters. Polymer 2005;46:4041-51.

6. Levchik SV, Weil ED. A review on thermal decomposition and combustion of thermoplastic polyesters. Polym Adv Technol 2004;15:691-700.

7. Jeong YG, Jo WH, Lee SC. Crystal structure of poly(hexamethylene 2,6- naphthalate). Polym J 2001;33:913-9.

8. Yun Y, Jin J, Lee M. J Polym Sci Polym Chem 1997;35:2777.

9. Wang JS Matyjaszewski K. Macromolecules 1995;28:7901.

10. Vasilieva YA, Thomas DB, Hennaux PE, McCormick CL. Polym Prepr 2003;44:886-7.

11. Cunningham MF. Living/controlled radical polymerizations in dispersed phase systems. Prog Polym Sci 2002;27:1039-67.

12. Kulinna C, Hendann C, Siesler HW. Selectively deuterated liquid crystalline cyanoazobenzene side-chain polyesters. 3 . Investigations of laser-induced segmental mobility by fourier transform infrared spectroscopy. Macromolecules 1999;31:2141.

13. Osswald AT, Menges G. Material Science of Polymers for Engineers. $3^{\text {rd }}$ ed. Germany: Hanser.

14. Fried RJ. Polymer Science and Technology. $3^{\text {rd }}$ ed. USA: Prentice Hall; 2014.

15. Billmeyer WF Jr. Textbook of Polymer Science. $3^{\text {rd }}$ ed. New York: Wiley; 2012.

16. Lussac JG, Pelouze. Neopenthyl glycol containing poly(propylene azelate)s: Synthesis and thermal properties. J Annu 1833;7:40.

17. Namkajora M, Petchsuk A. Effect of block length on solid-state properties and enzymatic degradation. Express Polym Lett 2010;4:415-22

18. Danuta S, Bozena K. The relation of transition temperatures to chemical structure in high polymers. Polymer 1998;39:3327-33.

19. Jackson WJ, KuhfussJ HF. Perspective: Comments on "liquid crystal polymers. I. Preparation and properties of p-hydroxybenzoic acid copolyesters. Polym Sci Polym Chem 1976;14:2043.

20. Suzuki K, Saito H. Biodegradable aliphatic polyesters. Part I. Properties and biodegradation of poly(butylene succinate-cobutylene adipate). Polymer 2005;46:8313-20.

21. Chang JH, Hwang BD. Synthesis and thermal behavior of thermotropic terpolymers based on 8-(3-hydroxyphenyl) octanoic acid, 2,6-naphthalenedicarboxylic acid, and substituted hydroquinones. Polymer 2002;43:2969-74.

22. Sanadhya SG. Parmar SL. Synthesis and characterization of aliphatic-aromatic polyesters using interfacial polycondensation technique. J Chem Pharm Res 2014;6:705-14

23. Sek D, Kaczmarczyk B. Effect of copolymerization in the dynamics of poly (trimethylene terephthalate). Polymer 1997;38:2925.

24. Sun S, Cao H, Su H. Tianwei tan synthesis bio-based terephthalate polyesters. Polym Bull 2009;23:66-74.

25. Middleton JC. ACS Div Polym Mater Sci Eng 1999;81:569.

26. Yang C, Xie H. Polymer 2013;54:67-77.
27. Bertini F, Zuev VV. Investigation on the thermal degradation of fully aromatic regular polyester. Poly Degrad Stab 2005;34:122-134.

28. Roviello A, Sirigu A. New versatile, elastomeric, degradable polymeric materials. Makromol Chem 1979;183:895.

29. Murray P, Whites J. ???. Trans Br Ceram Soc 1955;54:151.

30. Coats AW, Redfern JP. Kinetic parameters from thermogravimetric data. Nature 1964;201:68-9.

31. Doyle CD. Synthesis of novel compatibilisers and their application in PP/nylon-66 blends. I. Synthesis and characterization. J Appl Polym Sci 1962;6:639.

32. Gordon M, Plate NA. Liquid Crystal Polymers II/III. Berlin: Springer-Verlag; 1984.

33. Kelkar H. Synthesis, characterization and in vitro degradation of a novel degradable poly ((1,2-propanediol-sebacate)-citrate) bio elastomer. Cryst Liq Cryst 1979;21:1.

34. Gray GW. In: Cifferri A, Krigbaum WR, Meyer RB, editors. Polymer Liquid Crystals. New York: Academic Press; 1982.

35. Gray GW. In: Luckhurst GW, Gray GW, editors. The Molecular Physics of Liquid Crystals. New York: Academic Press; 1979.

36. Gray GW, Winsor PA. In: Gray GW, Winsor PA, editors. Liquid Crystals and Plastic Crystals. Vol. 1. England: Ellis Horwood Chichester; 1974.

37. Gray GW. In: Brown GH, editor. Advances in Liquid Crystals. Vol. 2. New York: Academic Press; 1976.

38. Wang X, Wang J. Appl Surf Sci 2010;256:183-195.

39. Windle AH, Viney C, Golombok R, Donald AM, Mitchell GR. Sequencesegregation in molten liquid crystalline random copolymers. Faraday Dis Chem Soc 1985;79:55.

40. Lin YG, Winter HH. Formation of a high melting crystal in a thermotropic aromatic copolyester. Macromolecules 1988;21:2439-43

41. Blundell DJ. The nature of crystallite in Solidified rigid-chain liquid crystal polymers. Polymer 1982;23:359.

42. Kaito A, Kyotani M, Nakayana K. Advances and applications of biodegradable elastomers in regenerative medicine. Macromolecules 1988;23:1035.

43. Cheng SZ. Kinetics of mesophase transitions in thermotropic polyesters. Macromolecules 1988;21:2475-84

Cite this article: Annamalai G, Subramanian $R$, Guhanathan S. Elango G. Synthesis and Spectral Characterization of New Aromatic Random Copolyester. Asian J Appl Res 2019;5(1):10-16.

Source of Support: Nil, Conflict of Interest: None declared. 